\title{
Strong trajectory statistical solutions and Liouville type equation for dissipative Euler equations*
}

\author{
Caidi Zhao ${ }^{a \dagger} \quad$ Zhongchun Song ${ }^{a \ddagger} \quad$ Tomás Caraballo ${ }^{b \S}$ \\ ${ }^{a}$ Department of Mathematics, Wenzhou University, \\ Wenzhou, Zhejiang Province, 325035, P. R. China \\ ${ }^{b}$ Departmento de Ecuaciones Diferenciales y Análisis Numérico \\ Facultad de Matemáticas, Universidad de Sevilla, \\ c/ Tarfia s/n, 41012-Sevilla, Spain
}

June 4, 2019

\begin{abstract}
The main aim of this letter is to use the strong compact strong trajectory attractor to construct the strong trajectory statistical solutions for two-dimensional dissipative Euler equations. Further, it is established that the constructed trajectory statistical solutions possess an invariant property and satisfy a Liouville type equation.

Keywords: Dissipative Euler equations; Strong trajectory statistical solution; Strong compact strong trajectory attractor; Liouville type equation;
\end{abstract}

MSC2010: 37L30; 35B41; 76F20

\section{Introduction}

In this letter, we study the so-called strong trajectory statistical solutions for the following two-dimensional (2D) damped Euler equations

$$
\left\{\begin{array}{l}
\frac{\partial u}{\partial t}+(u \cdot \nabla) u+R u+\nabla p=f \\
\nabla \cdot u=0,\left.u\right|_{t=0}=u_{0}
\end{array}\right.
$$

in the domain $\Omega=[-\pi, \pi]^{2}$ with periodic boundary conditions and with the external force $f \in W^{1, \infty}(\Omega)$. The equations in (1.1) describe a $2 \mathrm{D}$ fluid moving on a rough surface and are used in geophysical models for large scale processes in atmosphere and ocean. The term $R u$ parameterizes the main dissipation occurring in the planetary boundary layer. One can see [18], and also see [5] for the alternative source of the damped Euler equations.

Equations (1.1) and related ones are studied in a significan number of references, including analytic properties, stability analysis, vanishing viscosity limit and various attractors, see $[2$, $7,12,13,20]$. Especially, Chepyzhov, Vishik and Zelik in [7] proved the existence of a strong

*Supported by NSF of China with No.11271290 and by NSF of Zhejiang Province with No.LY17A010011. Also supported by FEDER and the Spanish Ministerio de Ciencia, Innovación y Universidades project PGC2018096540-I00.

${ }^{\dagger}$ Corresponding author E-mail: zhaocaidi2013@163.com or zhaocaidi@wzu.edu.cn

‡E-mail: mathzcs2013@163.com

§E-mail: caraball@us.es 
compact strong trajectory attractor for equations (1.1). The motivation of the current article is to construct the strong trajectory statistical solutions for equations (1.1).

There are two prevalent notions of statistical solutions. One is the so-called Foias-Prodi statistical solution introduced by Foias and Prodi in [8] and the other one is the so-called Vishik-Furshikov statistical solution given by Vishik and Furshikov in [19]. The Foias-Prodi statistical solutions are a family of Borel measures parametrized by the time variable and defined on the phase space of the Navier-Stokes equations, representing the probability distribution of the velocity field of the flow at each time. The Vishik-Furshikov statistical solutions are a single Borel measure on the space of trajectories, representing the probability distribution of the space-time velocity field.

The invariant measures for dissipative systems were studied in a series of references (see, for instance, [6,11,14-17]). More precisely, Łukaszewicz, Real and Robinson [17] used the notion of Generalized Banach limit to construct the invariant measures for general continuous dynamical systems on metric spaces. Later, Chekroun and Glatt-Holtz [6] improved the results of [17] to construct invariant measures for a broad class of dissipative autonomous dynamical systems. Recently, Łukaszewicz and Robinson [15] extended the result of [6] to construct invariant measures for dissipative non-autonomous dynamical systems. We recognize that both of the constructions of the invariant measures in [6] and [15] require that the addressed system is globally well-posed.

It is an interesting problem to construct invariant measures and trajectory statistical solutions for evolution equations which possess global weak solutions but without a known result of global uniqueness, say, the three-dimensional (3D) incompressible Navier-Stokes equations. In [3], Bronzi, Mondaini and Rosa proved a general framework for the theory of trajectory statistical solutions for evolution equations with similar properties to those of the 3D NavierStokes equations. In [4], Bronzi, Mondaini and Rosa established an abstract framework for the theory of statistical solutions for general evolution equations. We can find that the proofs in $[3,4]$ concerning the existence of trajectory statistical solutions for the initial value problem is based on the Krein-Milman approximation of the initial measure by convex combinations of Dirac deltas, as done in $[9,10]$. Very recently, Zhao and Caraballo used the weak trajectory attractor to construct the (weak) trajectory statistical solutions for the 3D globally modified Navier-Stokes equations in [21]. In the present letter, we will use the strong compact strong trajectory attractor to construct strong trajectory statistical solutions for the damped Euler equations (1.1).

\section{Strong trajectory statistical solutions and Liouville type equation}

We denote by $W^{m, p}(\Omega)=W^{m, p}, 1 \leqslant p \leqslant+\infty$, the Sobolev space of space-periodic functions whose distributional derivatives up to order $m$ belong to $L^{p}(\Omega)=L^{p}$, and $H^{m}=W^{m, 2}$ for short. We also set $\mathcal{V}=\left\{\varphi=\left(\varphi_{1}, \varphi_{2}\right) \in\left(\mathcal{C}_{0}^{\infty}(\Omega)\right)^{2} \mid \nabla \cdot \varphi=0\right\}$ and denote $\mathcal{H}^{m}=$ closure of $\mathcal{V}$ in $\left(H^{m}\right)^{2}$. Especially, $\mathcal{H}=\mathcal{H}^{0}$. The space $L_{\text {loc }}^{\infty}\left(\mathbb{R}_{+} ; \mathcal{H}^{1}\right)$ will be endowed with local weak-star topology $\Theta_{+}^{\mathrm{w}, \text { loc }}$ and strong topology $\Theta_{+}^{\mathrm{s}, \mathrm{loc}}$. By definition, a sequence $u_{n}(t) \longrightarrow u(t)(n \rightarrow \infty)$ in

the topology $\Theta_{+}^{\mathrm{w}, \text { loc }}$ (respectively $\Theta_{+}^{\text {s,loc }}$ ), if , for every $T>0, u_{n}(t) \rightarrow u(t)(n \rightarrow \infty)$ weakly-star in $L^{\infty}\left([0, T] ; \mathcal{H}^{1}\right)$, and respectively, $u_{n}(t) \longrightarrow u(t)(n \rightarrow \infty)$ strongly in $L^{\infty}\left([0, T] ; \mathcal{H}^{1}\right)$.

We set $\mathcal{F}_{+}^{b}:=L^{\infty}\left(\mathbb{R}_{+} ; \mathcal{H}^{1}\right)$ and let $\mathcal{K}_{+} \subset \mathcal{F}_{+}^{b}$ be the set of all solutions of (1.1) (see Definition 2.2 of [7]), and $T(t): \mathcal{K}_{+} \longmapsto \mathcal{K}_{+}, t \geqslant 0$ the translation semigroup $T(t) u(s):=u(t+s)$. For the definitions of strong compact strong trajectory attractor, we refer to [7].

Lemma 2.1. ( [7]) Let $f \in W^{1, \infty}(\Omega)$ and $R>0$.

(1) For any given $u_{0} \in \mathcal{H}^{1}$, there exists at least one solution $u$ of problem (1.1) .

(2) The damped Euler equations (1.1) possess a strong compact strong trajectory attractor $\mathcal{A}^{\text {tr }} \subset \mathcal{F}_{+}^{b}$. 
Notice that $\mathcal{F}_{+}^{b}$ is a Banach space. We endow the trajectory space $\mathcal{K}_{+} \subset \mathcal{F}_{+}^{b}$ with the metric $d_{\mathcal{K}_{+}}(u, v)=\sup _{t \in \mathbb{R}_{+}}\|u(t)-v(t)\|, u, v \in \mathcal{K}_{+}$. For a set $\mathcal{P} \subset \mathcal{K}_{+}$and some $\epsilon>0$, we denote $\mathcal{O}(\mathcal{P}, \epsilon)=\left\{v \in \mathcal{K}_{+} \mid d_{\mathcal{K}_{+}}(v, \mathcal{P})=\inf _{w \in \mathcal{P}} d_{\mathcal{K}_{+}}(v, w)<\epsilon\right\}$. In the sequel, we use $\mathcal{C}\left(\mathcal{K}_{+}\right)$to denote the set of continuous functions on $\mathcal{K}_{+}$.

We recall now two lemmas, which were proved in our recent article [21], since they play a crucial role to prove the existence of strong trajectory statistical solutions.

Lemma 2.2. ( [21]) Let $K$ be a compact subset of $\mathcal{K}_{+}$. Then, for every $\psi \in \mathcal{C}\left(\mathcal{K}_{+}\right)$, there exists some $\epsilon>0$ such that $\sup _{w \in \mathcal{O}(K, \epsilon)}|\psi(w)|<+\infty$.

Lemma 2.3. ( [21]) Let $K$ be a compact subset of $\mathcal{K}_{+}$and let $\psi, \phi \in \mathcal{C}\left(\mathcal{K}_{+}\right)$satisfy $\psi(w)=\phi(w)$ for every $w \in K$. Then, for every $\epsilon>0$, there exists a $\delta=\delta(\epsilon)>0$ such that $\sup _{w \in \mathcal{O}(K, \delta)} \mid \psi(w)-$ $\phi(w) \mid<\epsilon$.

We now state the definition of strong trajectory statistical solutions for the damped Euler equations (1.1).

Definition 2.1. A Borel probability measure $\rho$ on the trajectory space $\mathcal{K}_{+}$is said to be a strong $\mathcal{K}_{+}$-trajectory statistical solution over $\mathbb{R}_{+}$(or simply a strong trajectory statistical solution) for the damped Euler equations (1.1) if

(1) $\rho$ is tight for any $\mathcal{B} \in \mathcal{B}\left(\mathcal{K}_{+}\right)$(the collection of Borel sets of $\mathcal{K}_{+}$) in the sense that

$$
\rho(\mathcal{B})=\sup \left\{\rho(E) \mid E \in \mathcal{B}\left(\mathcal{K}_{+}\right) \text {and } E \subset \mathcal{B}\right\} ;
$$

(2) $\rho$ is supported by a Borel subset (w.r.t. the topology $\Theta_{+}^{\mathrm{s}, \text { loc }}$ ) of $\mathcal{K}_{+}$.

To construct the strong trajectory statistical solutions, we need the definition of generalized Banach limit (see $[9,15])$. Let $B_{+}$be the collection of all bounded real-valued functions on $\mathbb{R}_{+}$. For any generalized Banach limit $\operatorname{LIM}_{t \rightarrow+\infty}$, the following useful property

$$
\left|\operatorname{LIM}_{t \rightarrow+\infty} g(t)\right| \leqslant \limsup _{t \rightarrow+\infty}|g(t)|, \quad \forall g(\cdot) \in B_{+},
$$

is presented in $[9,(1.38)]$ and in $[6,(2.3)]$.

We now state and prove our main results. First, the existence of strong trajectory statistical solutions for the damped Euler equations (1.1) reads as follows.

Theorem 2.1. Let $f \in W^{1, \infty}(\Omega)$ and $R>0$. Then the damped Euler equations (1.1) possess at least one strong trajectory statistical solution which is supported by the strong compact strong trajectory attractor $\mathcal{A}^{\text {tr }}$, where the existence of $\mathcal{A}^{\text {tr }}$ is guaranteed by Lemma 2.1(2).

Proof. Let $\operatorname{LIM}_{t \rightarrow+\infty}$ be a given generalized Banach limit. Since $\mathcal{K}_{+}$is nonempty, we can pick some $v \in \mathcal{K}_{+}$. Consider some $\psi \in \mathcal{C}\left(\mathcal{K}_{+}\right)$. By Lemma 2.1(2), the translation semigroup $\{T(t)\}_{t \geqslant 0}$ possesses a strong compact strong trajectory attractor $\mathcal{A}^{\text {tr }} \subset \mathcal{K}_{+}$. By the attracting property of $\mathcal{A}^{\text {tr }}$ we see that for every $\epsilon>0$, there exists a time $t_{\epsilon} \geqslant 0$ such that

$$
T(t) v \in \mathcal{O}\left(\mathcal{A}^{\mathrm{tr}}, \epsilon\right) \text {, for every } t \geqslant t_{\epsilon} .
$$

By Lemma 2.2 we can choose $\epsilon>0$ such that

$$
C_{1}=\sup _{w \in \mathcal{O}\left(\mathcal{A}^{\mathrm{tr}}, \epsilon\right)}|\psi(w)|<+\infty .
$$

Since $T(t)$ maps $\mathcal{A}^{\text {tr }}$ continuously into itself, we see that the function $t \longmapsto|\psi(T(t) v)|$ is continuous on $\mathbb{R}_{+}$and thus $|\psi(T(t) v)|$ is bounded on the compact interval $\left[0, t_{\epsilon}\right]$. Hence, we can take $t_{\epsilon}$ as required in (2.2) for the picked $\epsilon$, and see that

$$
C_{2}=\sup _{t \in\left[0, t_{\epsilon}\right]}|\psi(T(t) v)|<+\infty .
$$


It then follows from (2.3) and (2.4) that

$$
\frac{1}{t} \int_{0}^{t} \psi(T(s) v) \mathrm{d} s=\frac{1}{t} \int_{0}^{t_{\epsilon}} \psi(T(s) v) \mathrm{d} s+\frac{1}{t} \int_{t_{\epsilon}}^{t} \psi(T(s) v) \mathrm{d} s \leqslant \frac{C_{2} t_{\epsilon}}{t}+\frac{C_{1}\left(t-t_{\epsilon}\right)}{t}<+\infty,
$$

which implies that the map defined by $t \longmapsto \frac{1}{t} \int_{0}^{t} \psi(T(s) v) \mathrm{d} s$ is bounded over $\mathbb{R}_{+}$. Therefore, if $\psi \in \mathcal{C}\left(\mathcal{K}_{+}\right)$is non-negative then

$$
\mathcal{L}_{v}(\psi):=\operatorname{LIM}_{t \rightarrow+\infty} \frac{1}{t} \int_{0}^{t} \psi(T(s) v) \mathrm{d} s
$$

is well defined as a positive linear functional on $\mathcal{C}\left(\mathcal{K}_{+}\right)$.

We next prove that the positive linear functional $\mathcal{L}_{v}(\psi)$ depends only on the values of $\psi$ on $\mathcal{A}^{\text {tr }}$. To this end, we shall prove that if $\psi(w)=\phi(w)$ for every $w \in \mathcal{A}^{\text {tr }}$ then $\mathcal{L}_{v}(\psi)=\mathcal{L}_{v}(\phi)$. Indeed, for any given $\epsilon>0$, we can choose, by Lemma 2.3 a corresponding $\delta>0$ such that

$$
\sup _{w \in \mathcal{O}\left(\mathcal{A}^{\operatorname{tr}}, \delta\right)}|\psi(w)-\phi(w)|<\epsilon .
$$

Let us now pick $t_{\delta}>0$ such that $T(t) v \in \mathcal{O}\left(\mathcal{A}^{\text {tr }}, \delta\right)$ for every $t \geqslant t_{\delta}$. Analogously to (2.4), we see that $C_{\delta}:=\sup _{t \in\left[0, t_{\delta}\right]}(|\psi(T(t) v)|+|\phi(T(t) v)|)<+\infty$. Combining (2.1), (2.4) and (2.6) yields

$\left|\mathcal{L}_{v}(\psi-\phi)\right|=\left|\operatorname{LIM}_{t \rightarrow+\infty} \frac{1}{t} \int_{0}^{t}(\psi(T(s) v)-\phi(T(s) v)) \mathrm{d} s\right| \leqslant \limsup _{t \rightarrow+\infty} \frac{t_{\delta} C_{\delta}}{t}+\limsup _{t \rightarrow+\infty} \frac{\left(t-t_{\delta}\right) \epsilon}{t} \leqslant \epsilon$.

Since $\epsilon>0$ is arbitrary, we obtain the desired result that the positive linear functional $\mathcal{L}_{v}(\psi)$ depends only on the values of $\psi$ on $\mathcal{A}^{\text {tr }}$.

Now we define $G(\psi)=\mathcal{L}_{v}(\widetilde{\psi})$, where $\widetilde{\psi}$ is a zero extension of $\psi$ from $\mathcal{C}\left(\mathcal{A}^{\text {tr }}\right)$ to $\mathcal{C}\left(\mathcal{K}_{+}\right)$given by the Tietze theorem (see [9, Theorem A.7]). We can find that $G(\cdot)$ is a positive linear functional on $\mathcal{C}\left(\mathcal{A}^{\text {tr }}\right)$. Notice that $\mathcal{A}^{\text {tr }} \subset \mathcal{K}_{+}$is compact w.r.t. the topology $\Theta_{+}^{\text {s,loc }}$. Then, obviously $\mathcal{A}^{\text {tr }}$ is a locally compact topological space w.r.t. the topology $\Theta_{+}^{\text {s,loc }}$. By the Kakutani-Riesz Representation Theorem (see [9, Theorem A.1]), we assert that there exists a unique positive, finite, Borel measure $\rho_{v}$ on $\mathcal{A}^{\text {tr }}$ such that

$$
G(\psi)=\int_{\mathcal{A}^{\operatorname{tr}}} \psi(u) \mathrm{d} \rho_{v}(u) .
$$

Taking $\rho_{v}(E)=\rho_{v}\left(\mathcal{A}^{\operatorname{tr}} \cap E\right)$ for $E \in \mathcal{B}\left(\mathcal{K}_{+}\right)$, we extend $\rho_{v}$ by zero to a Borel measure on $\mathcal{K}_{+}$. Thus for every $\psi \in \mathcal{C}\left(\mathcal{K}_{+}\right)$, we have

$$
G(\psi)=\mathcal{L}_{v}(\psi)=\operatorname{LIM}_{t \rightarrow+\infty} \frac{1}{t} \int_{0}^{t} \psi(T(s) v) \mathrm{d} s=\int_{\mathcal{A}^{\text {tr }}} \psi(u) \mathrm{d} \rho_{v}(u)=\int_{\mathcal{K}_{+}} \psi(u) \mathrm{d} \rho_{v}(u),
$$

and obviously $\rho_{v}\left(\mathcal{K}_{+} \backslash \mathcal{A}^{\text {tr }}\right)=0$. Since $\mathcal{K}_{+}$is contained in the Banach space $\mathcal{F}_{+}^{\mathrm{b}}, \mathcal{K}_{+}$is obviously a metrizable topological space, and thus every finite Borel measure in $\mathcal{K}_{+}$is tight in the sense of Definition 2.1(1) (see [1, Theorem 12.5]. To see that $\rho_{v}$ is a Borel probability measure we pick $\psi \equiv 1$ in (2.8). Therefore, by Definition 2.1, $\rho_{v}$ is a strong trajectory statistical solution for equations (1.1). The proof of Theorem 2.1 is completed.

We next establish that the trajectory statistical solution $\rho_{v}$ is invariant under the action of the translation semigroup $\{T(t)\}_{t \geqslant 0}$. Moreover, if we set $\mu_{t}=T(t) \rho_{v}$ defined by

$$
T(t) \rho_{v}(E)=\mu_{t}(E)=\rho_{v}\left(T(t)^{-1}(E)\right)
$$

for every set $E \subseteq \mathcal{K}_{+}$that is $\rho_{v}$-measurable, then we prove that $\mu_{t}$ satisfies a Liouville type equation in Statistical Mechanics. To this end, we need the definition and property of the class $\mathcal{T}$ of test functions on $\mathcal{H}$ (see [9, Chapter IV, Definition 1.2]). The definition of the class $\mathcal{T}$ of 
test functions on $\mathcal{H}$ are sufficient to ensure that if $u(t)$ solves equations (1.1) in the distributional sense, then

$$
\frac{\mathrm{d}}{\mathrm{d} t} \Phi(u(t))=\left\langle\Phi^{\prime}(u(t)), F(u(t))\right\rangle, \quad \forall \Phi \in \mathcal{T},
$$

where $F(u(t)):=P(f-(u \cdot \nabla) u+R u)$ and $P:\left(L^{2}\right)^{2} \mapsto \mathcal{H}$ is the Leray projection operator.

Theorem 2.2. (1) Let $\rho_{v}$ be the trajectory statistical solution proved in Theorem 2.1, then $\rho_{v}$ satisfies the following invariant property

$$
\int_{\mathcal{K}_{+}} \psi(T(t) u) \mathrm{d} \rho_{v}(u)=\int_{\mathcal{K}_{+}} \psi(u) \mathrm{d} \rho_{v}(u)=\int_{\mathcal{A}^{\text {tr }}} \psi(u) \mathrm{d} \rho_{v}(u), \quad \forall t \geqslant 0,
$$

for every $\psi \in \mathcal{C}\left(\mathcal{K}_{+}\right)$.

(2) $\mu_{t}=T(t) \rho_{v}$ satisfies the following Liouville type equation in Statistical Mechanics

$$
\frac{\mathrm{d}}{\mathrm{d} t} \int_{\mathcal{K}_{+}} \Phi(u) \mathrm{d} \mu_{t}(u)=\int_{\mathcal{K}_{+}}\left\langle\Phi^{\prime}(u), F(u)\right\rangle \mathrm{d} \mu_{t}(u),
$$

for all test functions $\Phi \in \mathcal{T}$.

Proof. We first prove that $\rho_{v}$ satisfies (2.11). To this end, we fix $t^{*} \geqslant 0$ and $\psi \in \mathcal{C}\left(\mathcal{K}_{+}\right)$. Since the interval $\left[0, t^{*}\right]$ is compact in $\mathbb{R}$ and $t \mapsto|\psi(T(t) u)|$ is continuous, we have, using the property of generalized Banach limit (2.1),

$$
\operatorname{LIM}_{t \rightarrow+\infty} \frac{1}{t} \int_{0}^{t^{*}} \psi(T(s) u) \mathrm{d} s=0 .
$$

At the same time, we use (2.3) and the property of generalized Banach limit (2.1) to deduce

$$
\operatorname{LIM}_{t \rightarrow+\infty} \frac{1}{t} \int_{t}^{t+t^{*}} \psi(T(s) u) \mathrm{d} s=0 .
$$

Therefore, (2.13) and (2.14) imply

$$
\begin{aligned}
& \int_{\mathcal{K}_{+}} \psi\left(T\left(t^{*}\right) u\right) \mathrm{d} \rho_{v}(u)=\operatorname{LIM}_{t \rightarrow+\infty} \frac{1}{t} \int_{0}^{t} \psi\left(T\left(t^{*}\right) T(s) u\right) \mathrm{d} s \\
= & \operatorname{LIM}_{t \rightarrow+\infty} \frac{1}{t} \int_{t}^{t+t^{*}} \psi(T(s) u) \mathrm{d} s-\operatorname{LIM}_{t \rightarrow+\infty} \frac{1}{t} \int_{0}^{t^{*}} \psi(T(s) u) \mathrm{d} s+\operatorname{LIM}_{t \rightarrow+\infty} \frac{1}{t} \int_{0}^{t} \psi(T(s) u) \mathrm{d} s \\
= & \operatorname{LIM}_{t \rightarrow+\infty} \frac{1}{t} \int_{0}^{t} \psi(T(s) u) \mathrm{d} s=\int_{\mathcal{K}_{+}} \psi(u) \mathrm{d} \rho_{v}(u) .
\end{aligned}
$$

The invariant property of $\rho_{v}$ under the action of $\{T(t)\}_{t \geqslant 0}$ is proved.

We next establish that $\mu_{t}=T(t) \rho_{v}$, defined by (2.9), satisfies the Liouville type equation in Statistical Mechanics. Indeed, since the mapping $T(t)$ from $\mathcal{K}_{+}$into itself is continuous and $\rho_{v}$ is a probability distribution on $\mathcal{K}_{+}$, it is not difficult to check that $\mu_{t}=T(t) \rho_{v}$ is a probability distribution on $\mathcal{K}_{+}$. Now, for any $\Phi \in \mathcal{T}$, we have

$$
\int_{\mathcal{K}_{+}} \Phi(u) \mathrm{d} \mu_{t}(u)=\int_{\mathcal{K}_{+}} \Phi(u) \mathrm{d}\left(T(t) \rho_{v}\right)(u)=\int_{\mathcal{K}_{+}} \Phi(T(t) u) \mathrm{d} \rho_{v}(u) .
$$

Notice that the function $t \longmapsto \Phi(T(t) u)$ is differentiable and (2.10) holds. We use the generalized chain differentiation rule to differentiate (2.15) and obtain

$$
\begin{aligned}
\frac{\mathrm{d}}{\mathrm{d} t} \int_{\mathcal{K}_{+}} \Phi(u) \mathrm{d} \mu_{t}(u) & =\frac{\mathrm{d}}{\mathrm{d} t} \int_{\mathcal{K}_{+}} \Phi(u) \mathrm{d}\left(T(t) \rho_{v}\right)(u)=\frac{\mathrm{d}}{\mathrm{d} t} \int_{\mathcal{K}_{+}} \Phi(T(t) u) \mathrm{d} \rho_{v}(u) \\
& =\int_{\mathcal{K}_{+}}\left\langle\Phi^{\prime}(u), F(u)\right\rangle \mathrm{d} \mu_{t}(u) .
\end{aligned}
$$

This proves (2.12). The proof of Theorem 2.2 is therefore completed. 
Remark 2.1. If statistical equilibrium has been reached by the system, then the statistical informations do not change with time, that is $\Phi^{\prime}(u(t))=0$. We point out that equation (2.11) describing the invariant property of the trajectory statistical solutions is a particular situation of the Liouville type equation (2.12) in the case $\Phi^{\prime}(u)=0$.

\section{References}

[1] C. D. Aliprentis, K. C. Border, Infinite Dimensional Analysis, A Hithhiker's Guide, third editon, Springer-Verlag, 2006.

[2] C.Bardos, E. S. Titi, Euler equations for incompressible ideal fluids, Russia Math. Surveys, 62(2007), 409-451.

[3] A. Bronzi, C. F. Mondaini, R. Rosa, Trajectory statistical solutions for three-dimensional Navier-Stokes-like systems, SIAM J. Math. Anal., 46(2014), 1893-1921.

[4] A. Bronzi, C. F. Mondaini, R. Rosa, Abstract framework for the theory of statistical solutions, J. Differential Equations, 260(2016), 8428-8484.

[5] S. Brull, L. Pareschi, Dissipative hydrodynamic models for the diffusion of impurities in a gas, Appl. Math. Lett., 19(2006), 516-521.

[6] M. Chekroun, N. E. Glatt-Holtz, Invariant measures for dissipative dynamical systems: Abstract results and applications, Comm. Math. Phys., 316(2012), 723-761.

[7] V. V. Chepyzhov, M. I. Vishik, S. V. Zelik, Strong trajectory attractor for dissipative Euler equations, J. Math. Pures Appl., 96(2011), 395-407.

[8] C. Foias, G. Prodi, Sur les solutions statistiques equations de Navier-Stokes, Ann. Math. Pura Appl., 111(1976), 307-330.

[9] C. Foias, O. Manley, R. Rosa, R. Temam, Navier-Stokes Equations and Turbulence, Cambridge University Press, Cambridge, 2001.

[10] C. Foias, R. Rosa, R. Temam, Properties of time-dependent statistical solutions of the three-dimensional Navier-Stokes equations, Annales de L'Institut Fourier, 63(2013), $2515-2573$.

[11] Cláudia B. Gentile Moussa, Invariant measures for multivalued semigroups, J. Math. Anal. Appl., 455(2017), 1234-1248.

[12] P.-L. Lions, Mathematical Topics in Fluid Mechanics, Vol. 1, Incompressible Models, Clarendon Press, Oxford, 1996.

[13] J.-L. Lions, Quelques méthodes de résolution des problèmes aux limites nonlinéaire, Dunod et Gauthier-Villars, Paris, 1969.

[14] X. Li, W. Shen, C. Sun, Invariant measures for complex-valued dissipative dynamical systems and applications, Discrete Cont. Dyn. Syst.-B, 22(2017), 2427-2446.

[15] G. Łukaszewicz, J. Robinson, Invariant measures for non-autonomous dissipative dynamical systems, Discrete Cont. Dyn. Syst., 34(2014), 4211-4222.

[16] G. Eukaszewicz, Pullback attractors and statistical solutions for 2-D Navier-Stokes equations, Discrete Cont. Dyn. Syst.-B, 9(2008), 643-659.

[17] G. Lukaszewicz, J. Real, J. C. Robinson, Invariant measures for dissipative dynamical systems and generalised Banach limits, J. Dyn. Differential Equations, 23(2011), 225-250.

[18] J. Pedlosky, Geophysical Fluid Dynamics, Springer, New York, 1979.

[19] M. I. Vishik, A. V. Fursikov, Translationally homogeous statistical solutions and individual solutions with infinite energy of a system of Navier-Stokes equations, Siberian Mathematical Journal, 19(1978), 710-729.

[20] R. Temam, Infinite Dimensional Dynamical Systems in Mechanics and Physics, Springer, New York, 1997.

[21] C. Zhao, T. Caraballo, Asymptotic regularity of trajectory attractor and trajectory statistical solution for the 3D globally modified Navier-Stokes equations, J. Differential Equations, 266(2019), 7205-7229. 